Analele Universităţii de Vest, Timişoara

Seria Matematică - Informatică

LIV , 2, (2016), 117- 130

\title{
Characterization of Dini Lipschitz Functions in Terms of Their Helgason Transform
}

Salah El Ouadih and Radouan Daher

\begin{abstract}
In this paper, using a generalized translation operator, we obtain an analog of Younis Theorem 5.2 in [6] for the Helgason Fourier transform of a set of functions satisfying the Dini Lipschitz condition in the space $L^{2}$ for functions on noncompact rank one Riemannian symmetric spaces.
\end{abstract}

AMS Subject Classification (2000). 42B37

Keywords. Symmetric space; Helgason Fourier transform; Dini Lipschitz condition; Generalized translation operator.

\section{Introduction}

Younis Theorem $5.2[6]$ characterized the set of functions in $L^{2}(\mathbb{R})$ satisfying the Cauchy Lipschitz condition by means of an asymptotic estimate growth of the norm of their Fourier transforms, namely we have:

Theorem 1.1. ([6]) Let $f \in L^{2}(\mathbb{R})$. Then the following are equivalents (i) $\quad\|f(x+t)-f(x)\|=O\left(\frac{t^{\delta}}{\left(\log \frac{1}{t}\right)^{\gamma}}\right)$, as $t \rightarrow 0,0<\delta<1, \gamma \geq 0$, (ii) $\quad \int_{|\lambda| \geq r}|\widehat{f}(\lambda)|^{2} d \lambda=O\left(\frac{r^{-2 \delta}}{(\log r)^{2 \gamma}}\right)$, as $r \rightarrow \infty$, where $\widehat{f}$ stands for the Fourier transform of $f$. 
In this paper, for rank one symmetric spaces, we prove the generalization of Theorem 1.1 for the Helgason Fourier transform of a class of functions satisfying the Dini Lipschitz condition in the space $L^{2}$. For this purpose, we use the generalized translation operator. We point out that similar results have been established in the context of non compact rank one Riemannian symetric spaces [9].

\section{Helgason Fourier Transformation on Symmetric Spaces}

Here we collect the necessary facts about the Fourier transformation on symmetric spaces and the spherical Fourier transformation (see $[1,2]$ ). For the required properties of semisimple Lie groups and symmetric spaces, we refer the reader, e.g., to $[3,4]$. An arbitrary Riemannian symmetric space $X$ of noncompact type can be represented as the factor space $G / K$, where $G$ is a connected noncompact semisimple Lie group with finite center, and $K$ is a maximal Compact subgroup of $G$. ON $X=G / K$ the group $G$ acts transitively by left shifts, and $K$ coincides with the stabilizer of the point $o=e K$ (e is the unity of $\mathrm{G}$ ). Let $G=N A K$ be an Iwasawa decomposition for $G$, and let $\mathfrak{g}, \mathfrak{k}, \mathfrak{a}, \mathfrak{n}$ be the Lie algebras of the groups $G, K, A, N$, respectively. We denote by $M$ we mean the centralizer of the subgroup $A$ in $K$ and put $B=K / M$. Let $d x$ be $a$ G-invariant measure on $X$; the symbols $d b$ and $d k$ will denote the normalized $K$-invariant measures on $B$ and $K$, respectively. We denote by $\mathfrak{a}^{*}$ the real space dual to $\mathfrak{a}$, and by $W$ the finite Weyl group acting on $\mathfrak{a}^{*}$. Let $\sum$ be the set of restricted roots $\left(\sum \subset \mathfrak{a}^{*}\right)$, Let $\sum^{+}$be the set of restricted positive roots, and let

$$
\mathfrak{a}^{+}=\left\{h \in \mathfrak{a}: \alpha(h)>0, \alpha \in \sum^{+}\right\},
$$

be the positive Weyl chamber. If $\rho$ is the half-sum of the positive roots (with multiplicity), then $\rho \in \mathfrak{a}^{*}$. Let $\langle$,$\rangle be the Killing form on the Lie$ algebra $\mathfrak{g}$. This form is positive definite on $\mathfrak{a}$. For $\lambda \in \mathfrak{a}^{*}$, let $H_{\lambda}$ denote a vector in $\mathfrak{a}$ such that $\lambda(H)=\left\langle H_{\lambda}, H\right\rangle$ for all $H \in \mathfrak{a}$. For $\lambda, \mu \in \mathfrak{a}^{*}$ we put $\langle\lambda, \mu\rangle:=\left\langle H_{\lambda}, H_{\mu}\right\rangle$. The correspondence $\lambda \mapsto H_{\lambda}$ enables us to identify $\mathfrak{a}^{*}$ and $\mathfrak{a}$. Via this identification, the action of the Weyl group $W$ can be transferred to $\mathfrak{a}$. Let

$$
\mathfrak{a}_{+}^{*}=\left\{\lambda \in \mathfrak{a}^{*}: H_{\lambda} \in \mathfrak{a}^{+}\right\} .
$$

If $X$ is a symmetric space of rank 1 , then $\operatorname{dim} \mathfrak{a}^{*}=1$, and the set $\sum^{+}$ consists of the roots $\alpha$ and $2 \alpha$ with some multiplicities $a$ and $b$ depending on 
$X$ (see [1]). In this case we identify the set $\mathfrak{a}^{*}$ with $\mathbb{R}$ via the correspondence $\lambda \leftrightarrow \lambda \alpha, \lambda \in \mathbb{R}$. Upon this identification positive numbers correspond to the set $\mathfrak{a}_{+}^{*}$. The numbers $m_{\alpha}$ and $m_{2 \alpha}$ are frequent in various formulas for rank 1 symmetric spaces. For example, the area of a sphere of radius $t$ on $X$ is equal to

$$
S(t)=c(\sinh t)^{m_{\alpha}}(\sinh 2 t)^{m_{2 \alpha}},
$$

where $c$ is some constant; the dimension of $X$ is equal to

$$
\operatorname{dim} X=m_{\alpha}+m_{2 \alpha}+1 .
$$

We return to the case in which $X=G / K$ is an arbitrary symmetric space. Given $g \in G$, denote by $A(g) \in \mathfrak{a}$ the unique element satisfying

$$
g=n \cdot \exp A(g) \cdot u
$$

where $u \in K$ and $n \in N$. For $x=g K \in X$ and $b=k M \in B=K / M$, we put

$$
A(x, b):=A\left(k^{-1} g\right) \text {. }
$$

We denote by $\mathcal{D}(X)$ and $\mathcal{D}(G)$ the sets of infinitely differentiable compactlysupported functions on $X$ and $G$. Let $d g$ be the element of the Haar measure on $G$. We assume that the Haar measure on $G$ is normed so that

$$
\int_{X} f(x) d x=\int_{G} f(g o) d g, \quad f \in \mathcal{D}(X) .
$$

For a function $f(x) \in \mathcal{D}(X)$, the Helgason Fourier transform on $X$ was introduced by S. Helgason (see [2] or [5]) and is defined by the formula

$$
\widehat{f}(\lambda, b):=\int_{X} f(x) e^{(i \lambda+\rho)(A(x, b))} d x, \lambda \in \mathfrak{a}^{*}, b \in B=K / M
$$

We can norm the measure on $X$ so that the inverse Fourier transform on $X$ would have the form

$$
f(x)=\frac{1}{|W|} \int_{\mathfrak{a}^{*} \times B} \widehat{f}(\lambda, b) e^{(i \lambda+\rho)(A(x, b))}|c(\lambda)|^{-2} d \lambda d b,
$$

where $|W|$ is the order of the Weyl group, $d \lambda$ is the element of the Euclidean measure on $\mathfrak{a}^{*}$, and $c(\lambda)$ is the Harish-Chandra function. Henceforth, for brevity, we use the notation

$$
d \mu(\lambda):=|c(\lambda)|^{-2} d \lambda
$$


Also, the Plancherel formula is valid:

$$
\|f\|_{2}^{2}:=\int_{X}|f(x)|^{2} d x=\frac{1}{|W|} \int_{\mathfrak{a}^{*} \times B}|\widehat{f}(\lambda, b)|^{2} d \mu(\lambda) d b=\int_{\mathfrak{a}_{+}^{*} \times B}|\widehat{f}(\lambda, b)|^{2} d \mu(\lambda) d b .
$$

By continuity, the mapping $f(x) \mapsto \widehat{f}(\lambda, b)$ extends from $\mathcal{D}(X)$ to an isomorphism of the Hilbert space $L^{2}(X)=L^{2}(X, d x)$ onto the Hilbert space $L^{2}\left(\mathfrak{a}_{+}^{*} \times B, d \mu(\lambda) d b\right)$.

Introduce the translation operator on $X$. Let $n=\operatorname{dim} X$. Denote by $d(x, y)$ the distance between points $x, y \in X$ and let

$$
\sigma(x ; t)=\{y \in X: d(x, y)=t\},
$$

be the sphere of radius $t>0$ on $X$ centered at $x$. Let $d \sigma_{x}(y)$ be the $(n-$ 1 )-dimensional area element of the sphere $\sigma(x ; t)$ and let $|\sigma(t)|$ be the area of the whole sphere $\sigma(x ; t)$ (it is independent of the point $x$ ). We denote by $C_{0}(X)$ the set of all continuous compactly-supported functions on $X$. Given $f(x) \in C_{0}(X)$, define the generalized translation operator $S^{h}$ by the formula

$$
\left(S^{t} f\right)(x)=\frac{1}{|\sigma(t)|} \int_{\sigma(x ; t)} f(y) d \sigma_{x}(y), t>0
$$

i.e., $\left(S^{t} f\right)(x)$ is the average of $f$ over $\sigma(x ; t)$.

Lemma 2.1. ([8]) The following inequality is valid for every function $f \in$ $L^{2}(X)$ and every $t \in \mathbb{R}_{+}=[0 ;+\infty)$ :

$$
\left\|S^{t} f\right\|_{2} \leq\|f\|_{2} \text {. }
$$

The polar decomposition of $G$ takes the form $G=K A^{+} K$, where $A^{+}=$ $\left\{a_{t}=\exp (A(g)): t \geq 0\right\}$. Following standard practice, functions $f$ on $X$ are identified with right $K$-invariant functions on $G$ and write $f(x)=f(g)$, where $x=g K$. In terms of this decomposition, the invariant measure $d x$ on $X$ has the form

$$
d x=\Delta(t) d t d k,
$$

where $\Delta(t)=\Delta_{(\alpha, \beta)}(t)=(2 \sinh t)^{2 \alpha+1}(2 \cosh t)^{2 \beta+1}, \alpha=\left(m_{\alpha}+m_{2 \alpha}-1\right) / 2$ and $\beta=\left(m_{2 \alpha}-1\right) / 2$, and $d k$ is normalized Haar measure on $K$. The Laplacian on $X$ is denoted $\Lambda$ and its radial part is given by

$$
\Lambda_{r}=\frac{d^{2}}{d t^{2}}+\frac{\Delta^{\prime}(t)}{\Delta(t)} \frac{d}{d t}
$$

The spherical function on $X$ is the unique radial solution to the equation

$$
\Lambda u=-\left(\lambda^{2}+\rho^{2}\right) u
$$


which is one at the origin of $X$.

An important role in harmonic analysis on symmetric spaces is played by spherical functions (see [1]). The Harish-Chandra formula for the spherical function is

$$
\varphi_{\lambda}(x)=\int_{B} e^{(i \lambda+\rho) A(x, b)} d b,
$$

where $d b$ is normalized measure on $B$. If we write $x=k a_{t} K$, then it is well known that $\varphi_{\lambda}(x)=\varphi_{\lambda}^{(\alpha, \beta)}(t)$, where $\varphi_{\lambda}^{(\alpha, \beta)}(t)$ is Jacobi function of the first kind (see $[10])$.

Lemma 2.2. ([8]) If $f \in L^{2}(X)$, then

$$
\widehat{S^{t} f}(\lambda, b)=\varphi_{\lambda}(t) \widehat{f}(\lambda, b), \quad \lambda, t \in \mathbb{R}_{+}=[0 ;+\infty) .
$$

Lemma 2.3. ([7]) The following inequalities are valid for a spherical function $\varphi_{\lambda}(t)\left(\lambda, t \in \mathbb{R}_{+}\right)$

(i) $\left|\varphi_{\lambda}(t)\right| \leq 1$,

(ii) $1-\varphi_{\lambda}(t) \leq t^{2}\left(\lambda^{2}+\rho^{2}\right)$,

(iii) there is a constant $c>0$ such that

$$
1-\varphi_{\lambda}(t) \geq c
$$

for $\lambda t \geq 1$.

Lemma 2.4. ([8]) Suppose that $f(x)$ and $\Lambda f(x)$ belong to $L^{2}(X)$. Then

$$
\widehat{\Lambda f}(\lambda, b)=-\left(\lambda^{2}+\rho^{2}\right) \widehat{f}(\lambda, b) .
$$

For $\alpha>\frac{-1}{2}$, we introduce the Bessel normalized function of the first kind $j_{\alpha}$ defined by

$$
j_{\alpha}(x)=\Gamma(\alpha+1) \sum_{n=0}^{\infty} \frac{(-1)^{n}(x / 2)^{2 n}}{n ! \Gamma(n+\alpha+1)}, \quad x \in \mathbb{R} .
$$

Moreover, we see that

$$
\lim _{x \rightarrow 0} \frac{j_{\alpha}(x)-1}{x^{2}} \neq 0
$$

Lemma 2.5. ([9, Lemma 9]) Let $\alpha \geq \beta \geq \frac{-1}{2}, \alpha \neq \frac{-1}{2}$ and let $t_{0}>0$. Then for $|\nu| \leq \rho$, there exists a positive constant $c_{0}$ such that

$$
\left|1-\varphi_{\lambda+i \nu}^{(\alpha, \beta)}(t)\right| \geq c_{0}\left|1-j_{\alpha}(\lambda t)\right|
$$

for all $0 \leq t \leq t_{0}$ 
For $f \in L^{2}(X)$, we define the finite differences of first and higher order as follows:

$$
\begin{aligned}
\Delta_{t}^{1} f & =\Delta_{t} f=\left(I-S^{t}\right) f, \\
\Delta_{t}^{k} f & =\Delta_{t}\left(\Delta_{t}^{k-1} f\right)=\left(I-S^{t}\right)^{k} f, \quad k=2,3, \ldots,
\end{aligned}
$$

where $I$ is the unit operator in the space $L^{2}(X)$.

We denote by $W^{2, k}, k \in \mathbb{N}^{*}$, the Sobolev space constructed by the operator $\Lambda$, i.e.,

$$
W^{2, k}=\left\{f \in L^{2}(X) ; \Lambda^{j} f \in L^{2}(X), j=0,1,2, \ldots, k\right\},
$$

where $\Lambda^{0} f=f, \Lambda^{1} f=\Lambda f, \Lambda^{m} f=\Lambda\left(\Lambda^{m-1} f\right), m=2,3, \ldots$.

\section{Dini Lipschitz Condition}

Definition 3.1. Let $f \in W^{2, k}$, and define

$$
\left\|\Delta_{t}^{k} \Lambda^{m} f\right\|_{2} \leq C \frac{t^{\eta}}{\left(\log \frac{1}{t}\right)^{\gamma}}, \quad \eta>0, \gamma \geq 0
$$

i.e.,

$$
\left\|\Delta_{t}^{k} \Lambda^{m} f\right\|_{2}=O\left(\frac{t^{\eta}}{\left(\log \frac{1}{t}\right)^{\gamma}}\right)
$$

for all $m=0,1, \ldots, k$, and for all sufficiently small $t, C$ being a positive constant. Then we say that $f$ satisfies a Dini Lipschitz of order $\eta$, or $f$ belongs to $\operatorname{Lip}(\eta, \gamma, 2)$.

Definition 3.2. If however

$$
\frac{\left\|\Delta_{t}^{k} \Lambda^{m} f\right\|_{2}}{\frac{t^{\eta}}{\left(\log \frac{1}{t}\right)^{\gamma}}} \rightarrow 0, \quad \text { as } \quad t \rightarrow 0
$$

i.e.,

$$
\left\|\Delta_{t}^{k} \Lambda^{m} f\right\|_{2}=O\left(\frac{t^{\eta}}{\left(\log \frac{1}{t}\right)^{\gamma}}\right),
$$

then $f$ is said to be belong to the little Dini Lipschitz class lip $(\eta, \gamma, 2)$.

Remark 3.1. It follows immediately from these definitions that

$$
\operatorname{lip}(\eta, \gamma, 2) \subset \operatorname{Lip}(\eta, \gamma, 2)
$$


Theorem 3.1. Let $\eta>1$. If $f \in \operatorname{Lip}(\eta, \gamma, 2)$, then $f \in \operatorname{lip}(1, \gamma, 2)$.

Proof. For $x \in X, t$ small and $f \in \operatorname{Lip}(\eta, \gamma, 2)$ we have

$$
\left\|\Delta_{t}^{k} \Lambda^{m} f\right\|_{2} \leq C \frac{t^{\eta}}{\left(\log \frac{1}{t}\right)^{\gamma}}
$$

Then

$$
\left(\log \frac{1}{t}\right)^{\gamma}\left\|\Delta_{t}^{k} \Lambda^{m} f\right\|_{2} \leq C t^{\eta}
$$

Therefore

$$
\frac{\left(\log \frac{1}{t}\right)^{\gamma}}{t}\left\|\Delta_{t}^{k} \Lambda^{m} f\right\|_{2} \leq C t^{\eta-1}
$$

which tends to zero with $t \rightarrow 0$. Thus

$$
\frac{\left(\log \frac{1}{t}\right)^{\gamma}}{t}\left\|\Delta_{t}^{k} \Lambda^{m} f\right\|_{2} \rightarrow 0, \quad t \rightarrow 0
$$

Then $f \in \operatorname{lip}(1, \gamma, 2)$.

Theorem 3.2. If $\eta<\nu$, then $\operatorname{Lip}(\eta, 0,2) \supset \operatorname{Lip}(\nu, 0,2)$ and $\operatorname{lip}(\eta, 0,2) \supset$ $\operatorname{lip}(\nu, 0,2)$.

Proof. We have $0 \leq t \leq 1$ and $\eta<\nu$, then $t^{\nu} \leq t^{\eta}$.

Then the proof of theorem is immediate.

\section{New Results On Dini Lipschitz Class}

Lemma 4.1. For $f \in W^{2, k}$, then

$$
\left\|\Delta_{t}^{k} \Lambda^{m} f\right\|_{2}^{2}=\int_{0}^{+\infty} \int_{B}\left(\lambda^{2}+\rho^{2}\right)^{2 m}\left|1-\varphi_{\lambda}(t)\right|^{2 k}|\widehat{f}(\lambda, b)|^{2} d \mu(\lambda) d b
$$

where $m=0,1, \ldots, k$.

Proof. Using Lemma 2.2 we get

$$
\begin{aligned}
\left.\widehat{\Delta_{t}^{1} f}(\lambda, b)\right) & =\widehat{f}(\lambda, b)-\widehat{S^{t} f}(\lambda, b) \\
& =\left(1-\varphi_{\lambda}(t)\right) \widehat{f}(\lambda, b),
\end{aligned}
$$

and

$$
\widehat{\Delta_{t}^{k}} f(\lambda, b)=\left(1-\varphi_{\lambda}(t)\right)^{k} \widehat{f}(\lambda, b)
$$


Furthermore, we obtain by the Lemma 2.4

$$
\widehat{\Lambda^{m}} f(\lambda, b)=(-1)^{m}\left(\lambda^{2}+\rho^{2}\right)^{m} \widehat{f}(\lambda, b) .
$$

Using the formulas (4.1) and (4.2) we get

$$
\widehat{\Delta_{t}^{k} \Lambda^{m}} f(\lambda, b)=(-1)^{m}\left(\lambda^{2}+\rho^{2}\right)^{m}\left(1-\varphi_{\lambda}(t)\right)^{k} \widehat{f}(\lambda, b) .
$$

Now by Plancherel formula, we have the result.

Theorem 4.2. Let $\eta>2 k$. If $f$ belong to the Dini Lipschitz class, i.e.,

$$
f \in \operatorname{Lip}(\eta, \gamma, 2), \quad \eta>2 k, \gamma \geq 0 .
$$

Then $f$ is the null function.

Proof. Assume that $f \in \operatorname{Lip}(\eta, \gamma, 2)$. Then

$$
\left\|\Delta_{t}^{k} \Lambda^{m} f\right\|_{2} \leq C \frac{t^{\eta}}{\left(\log \frac{1}{t}\right)^{\gamma}}, \quad \gamma \geq 0 .
$$

From Lemma 4.1, we have

$$
\int_{0}^{+\infty} \int_{B}\left(\lambda^{2}+\rho^{2}\right)^{2 m}\left|1-\varphi_{\lambda}(t)\right|^{2 k}|\widehat{f}(\lambda, b)|^{2} d \mu(\lambda) d b \leq C^{2} \frac{t^{2 \eta}}{\left(\log \frac{1}{t}\right)^{2 \gamma}} .
$$

In view of Lemma 2.5, we conclude that

$$
\int_{0}^{+\infty} \int_{B}\left(\lambda^{2}+\rho^{2}\right)^{2 m}\left|1-j_{\alpha}(\lambda t)\right|^{2 k}|\widehat{f}(\lambda, b)|^{2} d \mu(\lambda) d b \leq\left(\frac{C}{c_{0}}\right)^{2} \frac{t^{2 \eta}}{\left(\log \frac{1}{t}\right)^{2 \gamma}} .
$$

Then

$$
\frac{\int_{0}^{+\infty} \int_{B}\left(\lambda^{2}+\rho^{2}\right)^{2 m}\left|1-j_{\alpha}(\lambda t)\right|^{2 k}|\widehat{f}(\lambda, b)|^{2} d \mu(\lambda) d b}{t^{4 k}} \leq\left(\frac{C}{c_{0}}\right)^{2} \frac{t^{2 \eta-4 k}}{\left(\log \frac{1}{t}\right)^{2 \gamma}}
$$

Since $\eta>2 k$ we have

$$
\lim _{t \rightarrow 0} \frac{t^{2 \eta-4 k}}{\left(\log \frac{1}{t}\right)^{2 \gamma}}=0
$$

Then

$$
\lim _{t \rightarrow 0} \int_{0}^{+\infty} \int_{B}\left(\frac{\left|1-j_{\alpha}(\lambda t)\right|}{\lambda^{2} t^{2}}\right)^{2 k} \lambda^{4 k}\left(\lambda^{2}+\rho^{2}\right)^{2 m}|\widehat{f}(\lambda, b)|^{2} d \mu(\lambda) d b=0 .
$$

and also from the formula (2.1) and Fatou Theorem, we obtain

$$
\int_{0}^{+\infty} \int_{B} \lambda^{4 k}\left(\lambda^{2}+\rho^{2}\right)^{2 m}|\widehat{f}(\lambda, b)|^{2} d \mu(\lambda) d b=0 .
$$

and so $f$ is the null function. 
Analog of the Theorem 4.2, we obtain this Theorem.

Theorem 4.3. Let $f \in L^{2}(X)$. If $f$ belong to lip $(2,0,2)$. i.e.,

$$
\left\|\Delta_{t}^{k} \Lambda^{m} f\right\|_{2}=O\left(t^{2}\right), \quad \text { as } \quad t \rightarrow 0 .
$$

Then $f$ is the null function.

Now, we give another main result of this paper analog of Theorem 1.1.

Theorem 4.4. Let $f \in W^{2, k}$. Then the following are equivalents

(a) $f \in \operatorname{Lip}(\eta, \gamma, 2), \eta \in(0,1)$,

(b) $\int_{r}^{+\infty} \int_{B}\left(\lambda^{2}+\rho^{2}\right)^{2 m}|\widehat{f}(\lambda, b)|^{2} d \lambda d b=O\left(\frac{r^{-2 \eta-n+1}}{(\log r)^{2 \gamma}}\right)$, as $\quad r \rightarrow \infty$, where $m=0,1, \ldots, k$.

Proof. $(a) \Rightarrow(b)$ Let $f \in \operatorname{Lip}(\eta, \gamma, 2)$. Then we have

$$
\left\|\Delta_{t}^{k} \Lambda^{m} f\right\|_{2}^{2}=O\left(\frac{t^{\eta}}{\left(\log \frac{1}{t}\right)^{\gamma}}\right) \quad \text { as } \quad t \rightarrow 0 .
$$

From Lemma 3.2, we have

$$
\left\|\Delta_{t}^{k} \Lambda^{m} f\right\|_{2}^{2}=\int_{0}^{+\infty} \int_{B}\left(\lambda^{2}+\rho^{2}\right)^{2 m}\left|1-\varphi_{\lambda}(t)\right|^{2 k}|\widehat{f}(\lambda, b)|^{2} d \mu(\lambda) d b .
$$

If $\lambda \in\left[\frac{1}{t}, \frac{2}{t}\right]$, then $\lambda t \geq 1$ and (iii) of Lemma 2.3 implies that

$$
1 \leq \frac{1}{c^{2 k}}\left|1-\varphi_{\lambda}(t)\right|^{2 k}
$$

Then

$$
\begin{array}{r}
\int_{\frac{1}{t}}^{\frac{2}{t}} \int_{B}\left(\lambda^{2}+\rho^{2}\right)^{2 m}|\widehat{f}(\lambda, b)|^{2} d \mu(\lambda) d b \\
\leq \frac{1}{c^{2 k}} \int_{\frac{1}{t}}^{\frac{2}{t}} \int_{B}\left(\lambda^{2}+\rho^{2}\right)^{2 m}\left|1-\varphi_{\lambda}(t)\right|^{2 k}|\widehat{f}(\lambda, b)|^{2} d \mu(\lambda) d b \\
\leq \frac{1}{c^{2 k}} \int_{0}^{+\infty} \int_{B}\left(\lambda^{2}+\rho^{2}\right)^{2 m}\left|1-\varphi_{\lambda}(t)\right|^{2 k}|\widehat{f}(\lambda, b)|^{2} d \mu(\lambda) d b \\
\leq \frac{1}{c^{2 k}}\left\|\Delta_{t}^{k} \Lambda^{m} f\right\|_{2}^{2} \\
=O\left(\frac{t^{2 \eta}}{\left(\log \frac{1}{t}\right)^{2 \gamma}}\right) .
\end{array}
$$


From [8], we have

$$
|c(\lambda)|^{-2} \asymp \lambda^{n-1}, \quad n=\operatorname{dim} X,
$$

then

$$
\int_{\frac{1}{t}}^{\frac{2}{h}} \int_{B}\left(\lambda^{2}+\rho^{2}\right)^{2 m}|\widehat{f}(\lambda, b)|^{2} \lambda^{n-1} d \lambda d b=O\left(\frac{t^{2 \eta}}{\left(\log \frac{1}{t}\right)^{2 \gamma}}\right),
$$

or, equivalently,

$$
\int_{r}^{2 r} \int_{B}\left(\lambda^{2}+\rho^{2}\right)^{2 m}|\widehat{f}(\lambda, b)|^{2} d \lambda d b \leq C \frac{r^{-2 \eta-n+1}}{(\log r)^{2 \gamma}}, \quad r \rightarrow \infty,
$$

where $C$ is a positive constant. Now,

$$
\begin{array}{r}
\int_{r}^{+\infty} \int_{B}\left(\lambda^{2}+\rho^{2}\right)^{2 m}|\widehat{f}(\lambda, b)|^{2} d \lambda d b \\
=\sum_{i=0}^{\infty} \int_{2^{i} r}^{2^{i+1} r} \int_{B}\left(\lambda^{2}+\rho^{2}\right)^{2 m}|\widehat{f}(\lambda, b)|^{2} d \lambda d b \\
\leq C\left(\frac{r^{-2 \eta-n+1}}{(\log r)^{2 \gamma}}+\frac{(2 r)^{-2 \eta-n+1}}{(\log 2 r)^{2 \gamma}}+\frac{(4 r)^{-2 \eta-n+1}}{(\log 4 r)^{2 \gamma}}+\cdots\right) \\
\leq C \frac{r^{-2 \eta-n+1}}{(\log r)^{2 \gamma}}\left(1+2^{-2 \eta-n+1}+\left(2^{-2 \eta-n+1}\right)^{2}+\left(2^{-2 \eta-n+1}\right)^{3}+\cdots\right) \\
\leq K_{\eta, n} \frac{r^{-2 \eta-n+1}}{(\log r)^{2 \gamma}}
\end{array}
$$

where $K_{\eta, n}=C\left(1-2^{-2 \eta-n+1}\right)^{-1}$ since $2^{-2 \eta-n+1}<1$.

Consequently

$$
\int_{r}^{+\infty} \int_{B}\left(\lambda^{2}+\rho^{2}\right)^{2 m}|\widehat{f}(\lambda, b)|^{2} d \lambda d b=O\left(\frac{r^{-2 \eta-n+1}}{(\log r)^{2 \gamma}}\right), \quad \text { as } \quad r \rightarrow \infty .
$$

$(b) \Rightarrow(a)$. Suppose now that

$$
\int_{r}^{+\infty} \int_{B}\left(\lambda^{2}+\rho^{2}\right)^{2 m}|\widehat{f}(\lambda, b)|^{2} d \lambda d b=O\left(\frac{r^{-2 \eta-n+1}}{(\log r)^{2 \gamma}}\right), \quad \text { as } \quad r \rightarrow \infty
$$

then

$$
\int_{r}^{2 r} \int_{B}\left(\lambda^{2}+\rho^{2}\right)^{2 m}|\widehat{f}(\lambda, b)|^{2} d \lambda d b=O\left(\frac{r^{-2 \eta-n+1}}{(\log r)^{2 \gamma}}\right)
$$

whence

$$
\begin{aligned}
\int_{r}^{2 r} \int_{B}\left(\lambda^{2}+\rho^{2}\right)^{2 m}|\widehat{f}(\lambda, b)|^{2} \lambda^{n-1} d \lambda d b & \leq 2^{n-1} r^{n-1} \int_{r}^{2 r} \int_{B}\left(\lambda^{2}+\rho^{2}\right)^{2 m}|\widehat{f}(\lambda, b)|^{2} d \lambda d b \\
& \leq C^{\prime} \frac{r^{-2 \eta}}{(\log r)^{2 \gamma}}
\end{aligned}
$$


Now,

$$
\begin{aligned}
\int_{r}^{+\infty} \int_{B}\left(\lambda^{2}+\rho^{2}\right)^{2 m}|\widehat{f}(\lambda, b)|^{2} \lambda^{n-1} d \lambda d b & \leq \sum_{k=0}^{\infty} \int_{2^{k} r}^{2^{k+1} r} \int_{B}\left(\lambda^{2}+\rho^{2}\right)^{2 m}|\widehat{f}(\lambda, b)|^{2} \lambda^{n-1} d \lambda \\
& \leq C^{\prime} \sum_{k=0}^{\infty} 2^{-2 k \eta} \frac{r^{-2 \eta}}{(\log r)^{2 \gamma}} .
\end{aligned}
$$

Consequently,

$$
\int_{r}^{+\infty} \int_{B}\left(\lambda^{2}+\rho^{2}\right)^{2 m}|\widehat{f}(\lambda, b)|^{2} \lambda^{n-1} d \lambda d b=O\left(\frac{r^{-2 \eta}}{(\log r)^{2 \gamma}}\right),
$$

and, by $|c(\lambda)|^{-2} \asymp \lambda^{n-1}$,

$$
\int_{r}^{+\infty} \int_{B}\left(\lambda^{2}+\rho^{2}\right)^{2 m}|\widehat{f}(\lambda, b)|^{2} d \mu(\lambda) d b=O\left(\frac{r^{-2 \eta}}{(\log r)^{2 \gamma}}\right) .
$$

Write

$$
\left\|\Delta_{t}^{k} \Lambda^{m} f\right\|_{2}^{2}=I_{1}+I_{2}
$$

where

$$
I_{1}=\int_{0}^{\frac{1}{t}} \int_{B}\left(\lambda^{2}+\rho^{2}\right)^{2 m}\left|1-\varphi_{\lambda}(t)\right|^{2 k}|\widehat{f}(\lambda, b)|^{2} d \mu(\lambda) d b
$$

and

$$
I_{2}=\int_{\frac{1}{t}}^{+\infty} \int_{B}\left(\lambda^{2}+\rho^{2}\right)^{2 m}\left|1-\varphi_{\lambda}(t)\right|^{2 k}|\widehat{f}(\lambda, b)|^{2} d \mu(\lambda) d b .
$$

Firstly, it follows from the inequality $\left|\varphi_{\lambda}(t)\right| \leq 1$ that

$$
I_{2} \leq 2^{2 k} \int_{\frac{1}{t}}^{+\infty} \int_{B}\left(\lambda^{2}+\rho^{2}\right)^{2 m}|\widehat{f}(\lambda, b)|^{2} d \mu(\lambda) d b=O\left(\frac{t^{2 \eta}}{\left(\log \frac{1}{t}\right)^{2 \gamma}}\right), \quad \text { as } \quad t \rightarrow 0 .
$$

To estimate $I_{1}$, we use the inequalities $(i)$ and $(i i)$ of Lemma 2.3

$$
\begin{aligned}
I_{1} & =\int_{0}^{\frac{1}{t}} \int_{B}\left(\lambda^{2}+\rho^{2}\right)^{2 m}\left|1-\varphi_{\lambda}(t)\right|^{2 k-1}\left|1-\varphi_{\lambda}(t)\right||\widehat{f}(\lambda, b)|^{2} d \mu(\lambda) d b \\
& \leq\left. 2^{2 k-1} \int_{0}^{\frac{1}{t}} \int_{B}\left(\lambda^{2}+\rho^{2}\right)^{2 m}\left|1-\varphi_{\lambda}(t)\right| \widehat{f}(\lambda, b)\right|^{2} d \mu(\lambda) d b \\
& \leq 2^{2 k-1} t^{2} \int_{0}^{\frac{1}{t}} \int_{B}\left(\lambda^{2}+\rho^{2}\right)^{2 m+1}|\widehat{f}(\lambda, b)|^{2} d \mu(\lambda) d b .
\end{aligned}
$$


Now, we apply integration by parts for a function

$$
\phi(r)=\int_{r}^{+\infty} \int_{B}\left(\lambda^{2}+\rho^{2}\right)^{2 m}|\widehat{f}(\lambda, b)|^{2} d \mu(\lambda) d b,
$$

to get

$$
\begin{aligned}
I_{1} & \leq 2^{2 k-1} t^{2} \int_{0}^{1 / t}-\left(r^{2}+\rho^{2}\right) \phi^{\prime}(r) d r \\
& \leq 2^{2 k-1} t^{2} \int_{0}^{1 / t}-r^{2} \phi^{\prime}(r) d r \\
& \leq 2^{2 k-1} t^{2}\left(-\frac{1}{t^{2}} \phi\left(\frac{1}{t}\right)+2 \int_{0}^{1 / t} r \phi(r) d r\right) \\
& \leq-2^{2 k-1} \phi\left(\frac{1}{t}\right)+2^{2 k} t^{2} \int_{0}^{1 / t} r \phi(r) d r \\
& \leq 2^{2 k} t^{2} \int_{0}^{1 / t} r \phi(r) d r .
\end{aligned}
$$

Since $\phi(r)=O\left(\frac{r^{-2 \eta}}{(\log r)^{2 \gamma}}\right)$, we have $r \phi(r)=O\left(\frac{r^{1-2 \eta}}{(\log r)^{2 \gamma}}\right)$ and

$$
\begin{aligned}
\int_{0}^{1 / t} r \phi(r) d r & =O\left(\int_{0}^{1 / t} \frac{r^{1-2 \eta}}{(\log r)^{2 \gamma}} d r\right) \\
& =O\left(\frac{t^{2 \eta-1}}{\left(\log \frac{1}{t}\right)^{2 \gamma}} \int_{0}^{1 / t} d r\right) \\
& =O\left(\frac{t^{2 \eta-2}}{\left(\log \frac{1}{t}\right)^{2 \gamma}}\right)
\end{aligned}
$$

so that

$$
I_{1}=O\left(\frac{t^{2 \eta}}{\left(\log \frac{1}{t}\right)^{2 \gamma}}\right)
$$

Combining the estimates for $I_{1}$ and $I_{2}$ gives

$$
\left\|\Delta_{t}^{k} \Lambda^{m} f\right\|_{2}=O\left(\frac{t^{\eta}}{\left(\log \frac{1}{t}\right)^{\gamma}}\right) \quad \text { as } \quad t \rightarrow 0
$$

and this ends the proof of the theorem. 


\section{Acknowledgement}

The authors would like to thank the referee for his valuable comments and suggestions.

\section{References}

[1] S. Helgason, Groups and geometric analysis: Integral geometry, invariant differential operators, and Spherical Functions [Russian translation], Mir, Moscow, 1987

[2] S. Helgason, A duality for symmetric spaces with applications to group representations, Adv. Math., 5 (1), (1970), 1-154

[3] S. Helgason, Differential geometry and symmetric spaces [Russian translation], Mir, Moscow, 1964

[4] S. Helgason, Differential geometry, lie groups and symmetric spaces, Academic Press, New York, 1978

[5] S. Helgason, Geometric analysis on symmetric spaces, Providence, RI, 1994

[6] M. S. Younis, Fourier transforms of Dini-Lipschitz functions, Int. J. Math. Math. Sci., 9 (2), (1986), 301-312, doi:10.1155/S0161171286000376

[7] S. S. Platonov, Approximation of functions in $L_{2}$-metric on noncompact rank 1 symmetric space, Algebra Analiz., 11 (1), (1999), 244-270

[8] S. S. Platonov, The Fourier transform of function satisfying the Lipshitz condition on rank 1 symetric spaces, Siberian Math.J., 46 (2), (2005), 1108-1118

[9] W. O. Bray and M. A. Pinsky, Growth properties of Fourier transforms via module of continuity, Journal of Functional Analysis, 255 (288), 2256-2285

[10] T. H. Koornwinder, Jacobi functions and analysis on non-compact semisimple Lie groups, "Special Functions: Group Theoretical Aspects and Applications" (R. Askey, T.H. Koornwinder, and W. Schempp, Eds.), Reeidel, Dordrecht, 1984

Salah El Ouadih

Department of Mathematics, Faculty of Sciences Aïn Chock, University Hassan II Casablanca, Morocco

E-mail: salahwadih@gmail.com

Radouan Daher

Department of Mathematics, Faculty of Sciences Aïn Chock, University Hassan II Casablanca, Morocco

E-mail: rjdaher024@gmail.com

Received: 29.02.2016 
Accepted: 31.07.2016

Revised: 1.06.2016 